\title{
Mykologische Mittheilungen.
}

\author{
Von H. Zukal (Wien).
}

(Mit Tafel XI und XII.)

(Fortsetzung. ${ }^{1}$ )

Einige Mykologen werden vielleicht in dem eben erwähnten, kurzen, kolbigen Seitenzweige ein Archikarp erkennen; ich sehe dagegen in dem ganzen Vorgang nur eine bestimmte und sehr häufig auftretende Form der Verknäuelung, welche nicht einmal bei ein und derselben Species streng constant festgehalten wird. Nach durchgeführter Fächerung der bei der Knäuelbildung betheiligten Hyphen, erscheint das junge Perithecium anffallend grosszellig. Wenn dasselbe dann durch weiteres Wachsthum eine Grösse von $200-250 \mu$ erlangt und seine Rinde eine bereits tief schwarze Färbung angenommen hat, entsteht in seinem Inneren durch Verschleimung des, zu dieser Zeit dort vorhandenen, sehr zarten Pseudoparenchyms ein Hohlraum, während sich gleichzeitig in seinem basalen Theile ein Complex von dicken, glänzenden, reich mit Protoplasma und Fett erfüllten Hyphen (Ascogonen) bildet. Aus letzteren gehen aber nicht, wie in vielen anderen Fällen, die Asci als mehr oder minder directe Auszweigungen hervor, sondern die ascogenen Hyphen bilden zuerst ein zartes aber ziemlich mächtiges Pseudoparenchym und diesem entsprossen erst die Asci. Ob die Paraphysen aus demselben Gewebe hervorgehen, wie die Asci, konnte ich nicht ermitteln; übrigens sind die Paraphysen in unserem Falle so ephemerer Natur, dass sie gewöhnlich verschwunden sind, ehe noch die Sporenschläuche ihre volle Grösse erreicht haben. Die jungen Sporen erscheinen in dem auffallend dickwandigen Ascus in schief einreihiger Lage als gestreckt-elliptische Zellen und erhalten zuerst die Querwände, dann die Längswände. Bei ihrem weiteren Wachsthum kommen sie aus ihrer Lage und liegen im reifen Schlauche gewöhnlich unregelmässig zweireihig (13).

Bei der Reife springt das Perithecium am Scheitel unregelmässig in einigen grossen Lappen auf; einmal sah ich auch ein Perithecium in der Richtung eines Polarkreises regelmässig aufspringen und dann den obersten Theil der Perithecienwand, wie einen Deckel, abwerfen. Bei dem Aufreissen der Peritheciumwand spielt wahrscheinlich der sich ausdehnende Ascushaufen die active Rolle, während die Fruchtkörperwand, welche zu dieser Zeit nur noch aus abgestorbenen Zellen zusammengesetzt ist, passiv gedehnt wird und endlich an den Linien des geringsten Widerstandes reisst. Hier muss ich auch erwähnen, dass die Sporenschläuche nahezu gleichzeitig ausreifen und wahrscheinlich auch in sehr kurzen Intervallen die Sporen entleeren. Den Ejaculationsvorgang selbst habe ich zwar nicht beobachtet, aber ich fand in den Perithecien ent- 
weder sämmtliche Schläuche entleert oder alle noch mit Sporen erfüllt. Wie man aus den entleerten Schläuchen schliessen kann, wird bei der Ejaculation der oberste Theil des Ascus kappenartig abgeworfen und nach dem Entleerungsact der noch übrig bleibende Theil des Schlauches stark verkürzt.

Nun war noch der Beweis zu erbringen, dass die geschilderte Ascusfrucht mit der Stachybotrys lolulata in einem genetischen Zusammenhang stehe. Da handelte es sich vor Allem um reines Sporenmaterial. Behufs Gewinnung desselben wurden einige reife Perithecien zwischen zwei sterilisirten Uhrschälchen feucht gehaiten.

Binnen 3 Tagen hatten sämmtliche Fruchtkörper ihre Sporen ejaculirt und das obere Schälchen war mit den dort hingeschleuderten und festklebenden Sporen dicht bedeckt. Nun wurden die letzteren mittelst einer feinen Borste unter dem Simplex aufgehoben und einzeln in die Culturtropfen von 10 Objectträgern vertheilt. In jeden Culturtropfen, der aus destillirtem (ausgekochtem) Wasser bestand, war ausserdem eine Zahl ausgekochter Baumwollfasern gelegt worden. Fast sämmtliche Sporen keimten binnen 48 Stunden, doch war die Zahl der ausgesendeten Keimschläuche sehr verschieden (15). Sowie die Spitze eines Keimschlauches einen Baumwollfaden berührte, drang sie sofort in den letzteren ein, wobei sich der Faden in einer ganz auffallenden Weise verdünnte. Der eingedrungene Faden wäçhst gewöhnlich zunächst auf dem kürzesten Wege nach dem Zelllumen der. Wollfaser, um sich hier wieder zu verdicken und reichlich zu verästeln. Die Cellulose der Wollfäden scheint dem eindringenden Pilzmycel kaum einen nennenswerthen Widerstand entgegenzusetzen, sie wird augenscheinlich durch ein ausgeschiedenes knzym (?) chemisch gelöst und dann wahrscheinlich als Nahrungsstoff resorbirt. Nach 8 Tagen wurde von dem in die Wollfäden eingedrungenen Mycel die ersten Conidienträger aufgerichtet, dieselben waren jedoch so ausserordentlich zart und in allen Theilen so zwerghaft, dass ich Mühe hatte, mich von ihrer Identität mit der oben beschriebenen Stachybotrys lobulata zu überzeugen. Erst nach weiteren 10 Tagen traten solche Formen auf, die jeden Zweifel beseitigten. Mit ihrem Auftreten war auch der genetische Zusammenhang zwischen Stachybotrys lobulata und Cleistotheca papyrophila erwiesen. Nicht unerwähnt darf ich es lassen, dass sich auf einigen Objectträgern eigenthümliche höckerige Sclerotien bildeten, welche gewöhnlich die im Culturtropfen vorhandenen Wollfäden mit in ihren Gewebeverband aufnahmen. Da ich dieselben Sclerotien auch in Siachybotrys-Culturen, die ich auf schwedischem Filtrirpapier angelegt hatte, beobachtet habe, so hielt ich sie für wichtig genug, um sie besonders zu zeichnen. Wahrscheinlich gehören die schwärzlichen Knoten, die man in alten Schöpfpapieren häufig findet, auch hierher.

In technisch-praktischer Beziehung hat die obige Untersuchung zu folgenden Resultaten geführt: 
In den Baumwollballen, welche vor dem Verspinnen oft monatelang in den europäischen Magazinen lagern, treten oft missfarbige Stellen (die fleckigen Ketten der Baumwollspinner) auf, welche durch ein steriles Pilzmycel verursacht werden.

Ein Theil dieser Mycelien gehört sicherlich zu meiner Cleistotheca papyrophila. Die Conidienform des letzteren Pilzes, nämlich die Stachybotrys lobulata Berk. befällt aber nicht nur das Rohmaterial, sondern auch die aus letzterem erzeugten Industrieproducte, insbesondere das Papier. Der Erste, der darauf aufmerksam machte, dass die Papiere unter dem Angriff von Pilzmycelien leiden, war Wiesner. ${ }^{1}$ ) Ich selbst habe mich überzeugt, dass insbesondere Baumwollpapiere, wenn sie feucht gehalten werden, ein ausgezeichnetes Substrat für unseren Pilz abgeben. Das Papier wird an den von der Stachybotrys befallenen Stellen brüchig und zerfällt nach dem Trocknen wie Zunder. Hochinteressant ist es, dass unser Pilz auch in der gegenwärtig überall fabriksmässig erzeugten Holzcellulose als Schädling auftritt, welche Thatsache erst jüngst von $\mathrm{Höhnel}^{2}$ ) constatirt wurde.

Es können jedoch all diese Dinge, die Rohbaumwolle, Papiere, Manuscripte und die Holzcellulose relativ leicht vor unserem Pilz geschützt werden, durch Trockenheit und genügenden Luftzutritt.

\section{Lecythium. ${ }^{3}$ ) \\ nov. gen. Hypocreacearum.}

Ohne Stroma. Perithecien einzeln, weich, ganz oberflächlich, flaschenförmig, lebhaft blaugrün, mit langem Hals und deutlichem Ostiolum. Schläuche lineal, 8sporig, mit Paraphysen gemischt. Sporen spindelförmig, 4zellig, ungefärbt, an beiden Enden mit einem Anhängsel.

\section{Lecythium aerugineum ${ }^{4}$ ) nov. spec.}

(Taf. XI. Fig. 20-25.)

Ohne Stroma. Perithecien einzeln, weich, ganz oberflächlich, flaschenförmig (nämlich den altrömischen Thränenfläschchen ähnlich), aussen kleiig behaart, lebhaft blaugrün, mit langem Halse und deutlichem, von einem weissen Wimpernkranze umgebenen Ostiolum, 500 bis $800 \mu$ lang, in seinem bauchigen Theile etwa $150-200 \mu$ breit $(20,23,24)$.

1) Siehe Wiesner, Technische Mikroskopie 1869, p. 239 und dessen Mikroskopische Untersuchüng des Papieres, Wien 1887, p. 48.

$\left.{ }^{2}\right)$ Siehe v. Höhnel, Mittheilungen aus dem Laboratorium für technische Waarenkunde und Mikroskopie der k. k. technischen Hochschule in Wien. Centralorgan für Waarenkunde und Technologie, Heft 5.

s) Von $\tau \dot{b} \Lambda \eta \boldsymbol{\eta} v \dot{v} \iota v$, die Flasche.

4) aerugineum, nach Saccardo's Chromotaxia, tab. II, Nr. 37. 
Schläuche schmal lineal, langgestielt, oben abgestutzt, etwa $90-100 \mu$ lang (pars sporif.) und $6-7 \mu$ breit (21).

Paraphysen sehr dünn und zart, jedoch deutlich verzweigt (21).

Sporen zu 8, im Schlauche einreihig oder schief einreihig, spindelförmig, 4zellig, hyàlin, sehr zartwandig, an beiden Enden mit einem kegelförmigen, zelligen, leicht abfallenden Anhängsel versehen, etwa $20 \mu$ lang und $5-6 \mu$ breit. Anhängsel etwa $2-4 \mu$ lang (22).

Auf der Rinde alter Weidenbäume nächst dem Klopeiner See in Kärnthen, dann cultivirt in der feuchten Kammer. 1892.

Der Farbstoff dieses Ascomyceten wird durch die gewöhnlichen Mineralsäuren kaum verändert, von den Alkalien aber in das Gelbliche oder Gelblichbraune überführt. Alkohol und Aether lösen ihn nicht, ebensowenig kaltes Glycerin. Im heissen Glycerin wird er jedoch gelöst und in das Rauchgraue verfärbt. Genauere Untersuchungen über den Farbstoff konnten aus Mangel an Material nicht vorgenommen werden; doch scheint er nach Allem, was ich von ihm sah, eine grosse Aehnlichkeit mit dem Farbstoff von Chlorosplenium aeruginosum Oed., beziehungsweise von Mollisia Jungermanniae Nees zu besitzen. ${ }^{1}$ )

Sehr eigenthümlich, ja meines Wissens einzig dastehend, ist der Entwickelungsgang des Peritheciums. Dasselbe entsteht aus einem $50-80 \mu$ grossen, sphärischen Zellkörper, der blaugrün gefärbt und von einer deutlichen, pseudoparenchymatischen Rinde umgeben ist. Zahlreiche Rhizoiden befestigen dieses grüne Kügelchen an das Substrat, nämlich der Weidenrinde. Ueber die Entstehung des kugeligen Zellkörpers aus dem Mycel kann ich nichts mittheilen. W enn nun der erwähnte grüne Zellkörper eine gewisse Entwickelungsstufe erreicht hat, bricht er auf seinem Scheitel auf, und es wächst aus seiner Mitte ein Bündel oder Säulchen weisser, paralleler Hyphen in die Höhe (25).

Die mittleren Hyphen dieses Bündels wölben sich mit ihren Spitzen kuppelförmig zusammen, während sich die peripherischen wie in einer Garbe nach aussen neigen. Das aus dem sphärischen Zellkörper hervorwachsende Hyphenbündel bildet von allem Anfange an einen Hohlkegel, dessen Spitze dort liegt, wo sich die Hyphen kuppelförmig zusammenneigen. Dieser Hohlkegel wächst nun mit seinem Vegetationspunkte an der Spitze rasch in die Höhe, wobei in seinem oberen Theile die parallel neben einander fortlaufenden Hyphen in einer ähnlichen Weise mit einander verschmelzen, wie in den Hyphenpycniden von Fumago. ${ }^{2}$ ) Durch diese Verschmelzung

1) Bezüglich des Farbstoffes der beiden genannten Pilze verweise ich auf Rehm's Discomyceten in Rabenhorst's Krypt.-Flora, Pilze, 39. Lief., p. 754 . Nr. 7.

$\left.{ }^{2}\right)$ Siehe Zopf, Die Conidienfrüchte von Fumago. Nova Acta, Bd. 40, 


\section{$2 \mathrm{BHL}$ Biodiversity Heritage Library}

Zukal, Hugo. 1893. "Mykologische Mittheilungen." Oesterreichische botanische Zeitschrift 43(6), 211-215.

View This Item Online: https://www.biodiversitylibrary.org/item/89823

Permalink: https://www.biodiversitylibrary.org/partpdf/246874

\section{Holding Institution}

Missouri Botanical Garden, Peter H. Raven Library

\section{Sponsored by}

Missouri Botanical Garden

\section{Copyright \& Reuse}

Copyright Status: Public domain. The BHL considers that this work is no longer under copyright protection.

This document was created from content at the Biodiversity Heritage Library, the world's largest open access digital library for biodiversity literature and archives. Visit BHL at https://www.biodiversitylibrary.org. 